\title{
Common infection-related conditions and risk of lymphoid malignancies in older individuals
}

\author{
L A Anderson ${ }^{*}, 1$, A A Atman ${ }^{1}$, C M McShane ${ }^{1}$, G J Titmarsh ${ }^{1}$, E A Engels ${ }^{2}$ and J Koshiol ${ }^{2}$ \\ ${ }^{1}$ Centre for Public Health, Queen's University Belfast, Northern Ireland BT12 6BJ, UK and ${ }^{2}$ Division of Cancer Epidemiology and \\ Genetics, National Cancer Institute, National Institutes of Health, 9609 Medical Center Drive, MSC 9776, Bethesda, Maryland \\ 20892, USA
}

Background: Chronic antigenic stimulation may initiate non-Hodgkin (NHL) and Hodgkin lymphoma (HL) development. Antecedent, infection-related conditions have been associated, but evidence by lymphoproliferative subtype is limited.

Methods: From the US SEER-Medicare database, $44191 \mathrm{NHL}, 1832 \mathrm{HL}$ and 200000 population-based controls, frequencymatched to all SEER cancer cases, were selected. Logistic regression models, adjusted for potential confounders, compared infection-related conditions in controls with $\mathrm{HL}$ and NHL patients and by the NHL subtypes diffuse large B-cell, T-cell, follicular and marginal zone lymphoma (MZL). Stratification by race was undertaken.

Results: Respiratory tract infections were broadly associated with NHL, particularly MZL. Skin infections were associated with a $15-28 \%$ increased risk of $\mathrm{NHL}$ and with most NHL subtypes, particularly cellulitis with T-cell lymphoma (OR 1.36, 95\%Cl 1.24-1.49). Only herpes zoster remained associated with $\mathrm{HL}$ following Bonferroni correction (OR 1.55, 95\% Cl 1.28-1.87). Gastrointestinal and urinary tract infections were not strongly associated with $\mathrm{NHL}$ or $\mathrm{HL}$. In stratified analyses by race, sinusitis, pharyngitis, bronchitis and cellulitis showed stronger associations with total NHL in blacks than whites $(P<0.001)$.

Conclusions: Infections may contribute to the aetiologic pathway and/or be markers of underlying immune modulation. Precise elucidation of these mechanisms may provide important clues for understanding how immune disturbance contributes to lymphoma.

Chronic infections, including Epstein-Barr virus, human herpesvirus 8, human $\mathrm{T}$ lymphotropic virus type I, Plasmodium falciparum, hepatitis B virus, hepatitis C virus (HCV), Helicobacter pylori, Campylobacter jejuni, Chlamydia psittaci, Borrelia burgdorferi and human immunodeficiency virus, have been linked to the pathogenesis of non-Hodgkin lymphoma (NHL) (Hjalgrim and Engels, 2008), a heterogeneous group of disease entities characterised by the malignant transformation of B or T lymphocytes. Hodgkin lymphoma (HL), characterised by the presence of ReedSternberg cells, has been associated with Epstein-Barr virus, good hygiene and delayed exposure to infection (Serraino et al, 1991; Tavani et al, 2000).
Our immunological defence against pathogens utilises both the innate and adaptive immune systems. These systems working in synergy enable the host to clear infections. Genetic variation of genes involved in the innate immune response, including tumour necrosis factor receptor-associated factor, receptor-interacting serine-threonine kinase 3, BAT2, Toll-like receptor 6 (Cerhan et al, 2007) and Beta-Defensin 126 ( $\mathrm{Hu}$ et al, 2013), have been associated with an increased risk of NHL and have a role in infection recognition and control. Chronic antigenic stimulation has been postulated as a potential mechanism for lymphomagenesis (Hjalgrim and Engels, 2008), with acute, community-acquired infections potentially playing a role (Cartwright et al, 1988;

*Correspondence: Dr LA Anderson; Email: I.anderson@qub.ac.uk

Received 2 December 2013; revised 6 March 2014; accepted 8 March 2014; published online 1 April 2014 
La Vecchia et al, 1992; Tavani et al, 2000; Engels et al, 2004; Chang et al, 2005; Koshiol et al, 2011; Becker et al, 2012; Karunanayake et al, 2012; Liu et al, 2012).

Although one study of male US veterans reported associations with infections to be more profound in individuals aged $\leqslant 50$ years and those of black race (Koshiol et al, 2011); these results have not been replicated in other study populations. In addition, few studies have investigated infection-related conditions by NHL subtype (Anderson et al, 2009; Kristinsson et al, 2010). Using data from the Surveillance Epidemiology and End Results (SEER)-Medicare database, we previously reported an increased risk of chronic lymphocytic lymphoma (CLL), an NHL subtype, in patients with claims for respiratory and skin infections (Anderson et al, 2009). Similar associations have also been reported for lymphoplasmacytic lymphoma/Waldenström macroglobulinaemia using the US Veterans Affairs database (Kristinsson et al, 2010). Given the heterogeneous nature of NHLs and their disparate clinical and prognostic characteristics, the objective of the study was to investigate the role of infection-related conditions by additional NHL subtypes and HL in SEER-Medicare.

\section{MATERIALS AND METHODS}

Study design. The SEER-Medicare database links SEER and Medicare data (Anderson et al, 2008). Since 1973, the SEER program has collected demographic and clinical information on cancers diagnosed in multiple US sites and currently covers approximately $28 \%$ of the US population (SEER ${ }^{\star}$ Stat Databases (submitted November 2011)). Medicare provides federally funded health insurance for US citizens aged $\geqslant 65$ years, entitling them to Part A coverage for hospital inpatient care (Warren et al, 2002). Approximately $95 \%$ of beneficiaries subscribe to Part B coverage, including physician and outpatient services (Warren et al, 2002). Individuals may also subscribe to a health maintenance organisation (HMO) scheme providing capitated care; associated claims are not captured by Medicare (Engels et al, 2011).

We conducted a retrospective case-control study using SEERMedicare data. Cases were defined as individuals with a SEER diagnosis of a primary lymphoid malignancy between 1992 and 2005. In addition to overall NHL, we evaluated the main NHL subtypes, including diffuse large B-cell lymphoma (DLBCL), T-cell lymphoma, follicular lymphoma (FL) and marginal zone lymphoma (MZL). Other rarer subtypes and CLL/SLL, which has been previously reported (Anderson et al, 2009), were not reported seperately. HL cases were investigated although data on extranodal HL are not presented owing to the small number of cases $(N=83)$. Cancer case definition was based on the InterLymph hierarchical classification of lymphoid neoplasms (Turner et al, 2010). Cases were required to be aged 66-99 years at diagnosis of malignancy and to have at least 13 months of Part A and Part B and no HMO Medicare coverage before diagnosis to ensure sufficient time to accrue exposure status. Patients diagnosed with malignancy only at autopsy or by death certificate were excluded. Controls $(N=200000)$ were previously selected from the $5 \%$ random sample of all Medicare beneficiaries without cancer residing in SEER areas (Engels et al, 2011) and were frequency matched to a larger group of all cancer types in the SEER data set by gender, age (66-69, 70-74, 75-79, 80-84, 85-99 years) and year of selection.

To ascertain exposure to common infection-related conditions, Medicare hospital, physician and outpatient files were searched for claims made by the attending physicians for infections of the respiratory tract (including acute nasopharyngitis (common cold), bronchitis, pharyngitis, laryngitis, sinusitis, pneumonia), skin (cellulitis, herpes zoster), gastrointestinal tract (gingivitis/periodonitis, gastroenteritis) and urinary tract (acute pyelonephritis, cystitis, prostatitis). Infection-related conditions were selected if the prevalence in controls was $>0.5 \%$. Cases and controls with at least one claim for an infection were considered exposed. Claims occurring during the 13-month period before case diagnosis/ control selection were excluded to minimise the possibility that diagnoses were attributable to the disease process.

Statistical analysis. Polytomous logistic regression was used to calculate odds ratios (ORs) and 95\% confidence intervals (CIs) to assess the association of each infectious disease and subsequent risk of lymphoid malignancy subtypes compared with controls. In the variance computation for the ORs, the fact that each case subtype was compared with the same control population, that some controls later served as cases and that individuals could be selected more than once as controls were accommodated (Anderson et al, 2008).

The combined NHL analyses were stratified by white and black race. The Wald $P$-value for the interaction term between race and an infection was used to evaluate homogeneity between associations in whites and blacks. Stratified results for NHL subtypes, HL and/or other racial groups are not presented given the small sample sizes.

All analyses were adjusted for gender, age and year of diagnosis/ selection. No missing data were observed. As 112 main analyses were conducted to investigate the associations between each infectious disease and each lymphoid malignancy subtype, we used Bonferroni correction $(P<0.00045)$ to highlight associations (underlined) that remained significant after controlling for multiple comparisons.

To assess the time point at which infections may be important in the development of lymphoid malignancies, we investigated infections diagnosed at different time intervals preceding diagnosis (i.e., $\leqslant 30$ months, $31-48$ months, $49-72$ months, $>72$ months) for those that remained significant following Bonferroni correction. Analyses were conducted with and without patients with human immunodeficiency virus $(n=39)$; as similar point estimates were observed (data not shown), results are presented for the full cohort of patients.

\section{RESULTS}

There were small differences in the distribution of characteristics between the case and control groups due to non-restricted matching (Table 1). Compared with controls, NHL cases overall were less likely to be male and more likely to be older, of white race, selected in more recent calendar years and to have longer Medicare coverage. Conversely, HL cases appeared younger and had shorter Medicare coverage than controls.

As shown in Table 2, NHL patients were $10-17 \%$ more likely than controls to have had claims for respiratory tract infections, except common cold, $>13$ months before diagnosis (ORs 1.10-1.17). Skin infection claims, including those for cellulitis (OR 1.15, 95\% CI 1.12-1.18) and herpes zoster (OR 1.28, 95\% CI 1.22-1.35), and claims for prostatitis (OR 1.12, 95\% CI 1.07-1.17) were also more common in NHL cases overall than controls. MZL was most strongly associated with respiratory tract infections, including sinusitis, bronchitis, influenza and pneumonia, Table 2. Following adjustment for multiple comparisons, DLBCL, the most common NHL subtype, remained associated with sinusitis, bronchitis, influenza, cellulitis and herpes zoster, Table 2. FL was associated with claims for sinusitis, laryngitis and herpes zoster while T-cell lymphoma remained associated with pharyngitis and cellulitis only, Table 2. Herpes zoster was the only infection to remain significantly associated with HL following multiple comparison adjustment (OR 1.55; Table 2). 
Table 1. characteristics of controls and patients with non-Hodgkin lymphoma (overall and by subtype) and Hodgkin lymphoma

Non-Hodgkin lymphoma

\begin{tabular}{|c|c|c|c|c|c|c|c|}
\hline & $\begin{array}{c}\text { Controls } \\
(n=200000)\end{array}$ & $\begin{array}{c}\text { Overall } \\
(n=44191)\end{array}$ & $\begin{array}{c}\text { Diffuse large B-cell } \\
\text { lymphoma } \\
(n=15883)\end{array}$ & $\begin{array}{c}\text { T-cell } \\
\text { non-Hodgkin } \\
\text { lymphoma } \\
(n=2813)\end{array}$ & $\begin{array}{l}\text { Follicular } \\
\text { lymphoma } \\
(n=4491)\end{array}$ & $\begin{array}{l}\text { Marginal zone } \\
\text { lymphoma } \\
(n=3223)\end{array}$ & $\begin{array}{l}\text { Hodgkin } \\
\text { lymphoma } \\
(n=1832)\end{array}$ \\
\hline \multicolumn{8}{|l|}{ Gender } \\
\hline $\begin{array}{l}\text { Male } \\
\text { Female }\end{array}$ & $\begin{array}{r}106172(53.0 \%) \\
93828(47.0 \%)\end{array}$ & $\begin{array}{l}20475 \text { (46.3\%) } \\
23716 \text { (53.7\%) }\end{array}$ & $\begin{array}{l}7184(45.2 \%) \\
8699 \text { (54.8\%) }\end{array}$ & $\begin{array}{l}1544(54.9 \%) \\
1269(45.1 \%)\end{array}$ & $\begin{array}{l}3122(43.0 \%) \\
4142(57.0 \%)\end{array}$ & $\begin{array}{l}1334(41.4 \%) \\
1889(58.6 \%)\end{array}$ & $\begin{array}{l}874(47.7 \%) \\
958 \text { (52.3\%) }\end{array}$ \\
\hline \multicolumn{8}{|l|}{ Age (years) } \\
\hline $\begin{array}{l}66-69 \\
70-74 \\
75-79 \\
80-84 \\
85-99\end{array}$ & $\begin{array}{l}33780 \text { (16.9\%) } \\
52008 \text { (26.0\%) } \\
50440 \text { (25.2\%) } \\
36097(18.0 \%) \\
27675(13.8 \%)\end{array}$ & $\begin{array}{r}6542(14.8) \\
10594(24.0 \%) \\
11233(25.4 \%) \\
8855(20.4 \%) \\
6967(15.8 \%)\end{array}$ & $\begin{array}{l}2110(13.3 \%) \\
3679(23.2 \%) \\
4099(25.8 \%) \\
3297(20.8 \%) \\
2698(17.0 \%)\end{array}$ & $\begin{array}{l}442(15.7 \%) \\
770(27.4 \%) \\
724(25.7 \%) \\
512(18.2 \%) \\
365(13.0 \%)\end{array}$ & $\begin{array}{r}1390(19.1 \%) \\
1941(26.7 \%) \\
1819(25.0 \%) \\
1309(18.0 \%) \\
805(11.1 \%)\end{array}$ & $\begin{array}{l}489(15.2 \%) \\
776(24.1 \%) \\
788(24.5 \%) \\
676(21.0 \%) \\
494(15.3 \%)\end{array}$ & $\begin{array}{l}337(18.4 \%) \\
486(26.5 \%) \\
469(25.6 \%) \\
318(17.4 \%) \\
222(12.1 \%)\end{array}$ \\
\hline \multicolumn{8}{|l|}{ Race } \\
\hline $\begin{array}{l}\text { White } \\
\text { Black } \\
\text { Asian } \\
\text { Hispanic } \\
\text { Other/unknown }\end{array}$ & $\begin{array}{c}166827(83.3 \%) \\
13949(7.0 \%) \\
8097(4.0 \%) \\
5199(2.6 \%) \\
5927(3.0 \%)\end{array}$ & $\begin{array}{c}39300 \text { (88.9\%) } \\
1823(4.1 \%) \\
1146(2.6 \%) \\
774(1.8 \%) \\
1148(2.6 \%)\end{array}$ & $\begin{array}{c}14042(88.4 \%) \\
536(3.4 \%) \\
457(2.9 \%) \\
349(2.2 \%) \\
499(3.1 \%)\end{array}$ & $\begin{array}{c}2345(83.4 \%) \\
207(7.4 \%) \\
117(4.2 \%) \\
46(1.6 \%) \\
98(3.5 \%)\end{array}$ & $\begin{array}{c}6681(92.0 \%) \\
221(3.0 \%) \\
120(1.7 \%) \\
102(1.4 \%) \\
140(1.9 \%)\end{array}$ & $\begin{array}{r}2800 \text { (86.9\%) } \\
172(5.3 \%) \\
108(3.4 \%) \\
65(2.0 \%) \\
78(2.4 \%)\end{array}$ & $\begin{array}{r}1632(89 \%) \\
76(4.2 \%) \\
28(1.5 \%) \\
50(2.7 \%) \\
46(2.5 \%)\end{array}$ \\
\hline \multicolumn{8}{|c|}{ Year of selection } \\
\hline $\begin{array}{l}1992-1994 \\
1995-1998 \\
1999-2005\end{array}$ & $\begin{array}{r}31364(15.7 \%) \\
39843(19.9 \%) \\
128793(64.4 \%)\end{array}$ & $\begin{array}{r}5930(13.4 \%) \\
8665(19.6 \%) \\
29596(67.0 \%)\end{array}$ & $\begin{array}{r}2063(13.0 \%) \\
3044(19.2 \%) \\
10776(67.9 \%)\end{array}$ & $\begin{array}{r}323(11.5 \%) \\
589(20.9 \%) \\
1901(67.6 \%)\end{array}$ & $\begin{array}{r}962(13.2 \%) \\
1351(18.6 \%) \\
4951(68.2 \%)\end{array}$ & $\begin{array}{r}37(1.15 \%) \\
519(16.1 \%) \\
2667(82.8 \%)\end{array}$ & $\begin{array}{r}275(15 \%) \\
330(18 \%) \\
1227(67 \%)\end{array}$ \\
\hline \multicolumn{8}{|c|}{ Duration of Medicare coverage (months) } \\
\hline $\begin{array}{l}13-60 \\
61-120 \\
121-180 \\
181-240\end{array}$ & $\begin{array}{c}57440(28.7 \%) \\
97485(48.7 \%) \\
35805(17.9 \%) \\
9270(4.6 \%)\end{array}$ & $\begin{array}{c}11330(25.6 \%) \\
21798(49.3 \%) \\
8699(19.7 \%) \\
2364(5.4 \%)\end{array}$ & $\begin{array}{c}3815(24.0 \%) \\
7951(50.1 \%) \\
3212(20.2 \%) \\
905(5.7 \%)\end{array}$ & $\begin{array}{c}775(27.6 \%) \\
1376(48.9 \%) \\
534(19.0 \%) \\
128(4.6 \%)\end{array}$ & $\begin{array}{c}2159(29.7 \%) \\
3503(48.2 \%) \\
1276(17.6 \%) \\
326(4.5 \%)\end{array}$ & $\begin{array}{r}893(27.7 \%) \\
1514(47.0 \%) \\
612(19.0 \%) \\
204(6.3 \%)\end{array}$ & $\begin{array}{c}555(30.3 \%) \\
911(49.7 \%) \\
296(16.2 \%) \\
70(3.8 \%)\end{array}$ \\
\hline
\end{tabular}

In stratified analyses by race, associations among whites were similar to those all subjects combined for most infection-related conditions, due to the majority of the cohort being of white race (Tables 2 and 3). For blacks, the only infection-related conditions to reach statistical significance following Bonferroni correction were sinusitis, pharyngitis and cellulitis. However, several infections, including sinusitis, pharyngitis, bronchitis and cellulitis, were more commonly associated with NHL in blacks than in whites, $P<0.001$.

Most infections highly significant in Table 2 remained associated with NHL even when the 6-year period preceding diagnosis was excluded (Table 4). Sinusitis, bronchitis, cellulitis and herpes zoster remained associated with DLBCL while cellulitis was associated with T-cell NHL across all time periods investigated (Table 4). For FL, sinusitis, laryngitis and herpes zoster were significant at longer latencies. Sinusitis was associated with MZL across all time periods investigated (Table 4). Claims for herpes zoster $>30$ months before diagnosis were associated with HL (Table 4).

\section{DISCUSSION}

In the largest population-based study to date, we identified respiratory and skin infections to be associated with an increased risk of NHL in individuals aged $\geqslant 66$ years, paralleling our previous findings for CLL (Anderson et al, 2009). MZLs were associated with the broadest range of infection-related conditions and T-cell lymphomas the fewest. Interestingly, only herpes zoster infection was associated with $\mathrm{HL}$, an association which remained even when initial claims were made $>6$ years before cancer diagnosis.

Consistent with the observed association between respiratory infections and NHL, Koshiol et al (2011) reported an increased risk of NHL among male US Veterans with upper and lower airway infections, including sinusitis and pneumonia, but did not report by NHL subtype. They identified stronger associations close to NHL diagnosis and postulated that this may have been due to reverse causality due to an underlying, undetected NHL (Koshiol et al, 2011; Richardson et al, 2011). In the current investigation, most associations occurring close to diagnosis were for MZL, an indolent, slow growing lymphoma, potentially supporting this hypothesis. Koshiol et al (2011) also suggested that undetected lymphoma would not fully explain why several infection-related conditions, including lower airway infections, occurred more frequently in cases $>5$ years before diagnosis. This observation was also apparent in the current study and was particularly evident for DLBCL and FL. Most FL cases have t $(14 ; 18)$-positive B cells, which are thought to be transformed by exogenous antigen stimulation, such as from a viral infection (Roulland et al, 2006). Antigenic 


\begin{tabular}{|c|c|c|c|c|c|c|c|c|c|c|c|c|c|}
\hline \multirow[b]{3}{*}{$\begin{array}{l}\text { Infection- } \\
\text { related } \\
\text { conditions by } \\
\text { site }\end{array}$} & \multirow{3}{*}{\begin{tabular}{|c|} 
Controls \\
No.
\end{tabular}} & \multirow{2}{*}{\multicolumn{2}{|c|}{$\begin{array}{l}\text { Overall NHL } \\
(n=44191)\end{array}$}} & \multirow{2}{*}{\multicolumn{2}{|c|}{$\begin{array}{l}\text { Diffuse large } \\
\text { B- cell lymphoma } \\
(n=15883)\end{array}$}} & \multicolumn{4}{|c|}{ NHL subtypes } & \multirow{2}{*}{\multicolumn{2}{|c|}{$\begin{array}{l}\text { Marginal zone } \\
\text { lymphoma } \\
(n=3223)\end{array}$}} & \multirow{2}{*}{\multicolumn{2}{|c|}{$\begin{array}{l}\text { Hodgkin } \\
\text { lymphoma } \\
(n=1832)\end{array}$}} \\
\hline & & & & & & \multicolumn{2}{|c|}{$\begin{array}{l}\text { T-cell NHL } \\
(n=2813)\end{array}$} & \multicolumn{2}{|c|}{$\begin{array}{l}\text { Follicular } \\
\text { lymphoma } \\
(n=4491)\end{array}$} & & & & \\
\hline & & No. & $\begin{array}{c}\text { OR } \\
(95 \% \mathrm{Cl})^{a}\end{array}$ & No. & $\begin{array}{c}\text { OR } \\
(95 \% \mathrm{Cl})^{\mathrm{a}}\end{array}$ & No. & $\begin{array}{c}\text { OR } \\
(95 \% \mathrm{Cl})^{a}\end{array}$ & No. & $\begin{array}{c}\text { OR } \\
(95 \% \mathrm{Cl})^{a}\end{array}$ & \multicolumn{2}{|r|}{$\begin{array}{c}\text { OR } \\
(95 \% \mathrm{Cl})^{\mathrm{a}}\end{array}$} & No. & $\begin{array}{c}\text { OR } \\
(95 \% \mathrm{Cl})^{a}\end{array}$ \\
\hline \multicolumn{14}{|c|}{ Respiratory tract } \\
\hline Common cold & 7322 & 1801 & $1.06(1.00-1.12)$ & 624 & $1.00(0.92-1.09)$ & 119 & $1.12(0.93-1.35)$ & 279 & $\mid 1.02(0.91-1.16)$ & 171 & $1.24(1.06-1.45)$ & 66 & $0.97(0.76-1.25)$ \\
\hline Sinusitis & 36249 & 9464 & $1.17(1.13-1.20)$ & 3389 & $1.14(1.09-1.19)$ & 544 & $1.04(0.94-1.14)$ & 1560 & $1.16(1.10-1.24)$ & 853 & $1.32(1.21-1.43)$ & 380 & $1.15(1.02-1.29)$ \\
\hline Laryngitis & 6584 & 1810 & $1.17(1.11-1.24)$ & 652 & $\overline{1.15}(1.06-1.25)$ & 119 & $1.26(1.04-1.51)$ & 310 & $1.25(1.11-1.41)$ & 157 & $1.23(1.05-1.45)$ & 75 & $1.23(0.97-1.55)$ \\
\hline Pharyngitis & 22472 & 5695 & $\overline{1.11}(1.07-1.15)$ & 1959 & $1.03(0.98-1.09)$ & 396 & $1.25(1.12-1.39)$ & 915 & $\overline{1.09}(\overline{1.01}-\overline{1.17)}$ & 476 & $1.14(1.03-1.26)$ & 240 & $1.17(1.02-1.34)$ \\
\hline Bronchitis & 45215 & 11458 & $\overline{1.13}(1.10-1.16)$ & 4123 & $1.11(1.06-1.15)$ & 704 & $\overline{1.10}(\overline{1.01}-\overline{1.21)}$ & 1779 & $1.08(1.02-1.15)$ & 939 & $1.15(1.07-1.25)$ & 446 & $1.09(0.98-1.22)$ \\
\hline Influenza & 14726 & 3892 & $\overline{1.15}(1.11-1.19)$ & 1389 & $\overline{1.11}(1.05-1.18)$ & 255 & $1.21(1.06-1.38)$ & 628 & $1.16(1.06-1.26)$ & 370 & $\overline{1.33}(1.19-\overline{1.49)}$ & 157 & $1.17(0.99-1.38)$ \\
\hline Pneumonia & 30201 & 7556 & 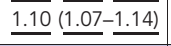 & 2679 & $\overline{1.05}(\overline{1.01}-1.10)$ & 448 & $1.04(0.94-1.16)$ & 1093 & $1.03(0.96-1.10)$ & 633 & $\overline{1.22}(1.11-\overline{1.34)}$ & 280 & $1.05(0.92-1.19)$ \\
\hline \multicolumn{14}{|l|}{ Skin } \\
\hline Cellulitis & 34426 & 8939 & $1.15(1.12-1.18)$ & 3176 & $1.09(1.05-1.14)$ & 627 & 1.36 (1.24-1.49) & 1352 & $1.10(1.04-1.18)$ & 751 & 1.23 (1.13-1.34) & 345 & $1.13(1.00-1.28)$ \\
\hline Herpes zoster & 8557 & 2553 & $\overline{1.28}(\overline{(1.22}-\overline{1.35)}$ & 889 & $\overline{1.20} \underline{(1.12}-\overline{1.30)}$ & 153 & $\overline{1.25} \overline{(1.06}-\overline{1.48)}$ & 386 & $1.24 \underline{(1.11-1.38)}$ & 200 & $\overline{1.22}(\overline{1.06}-\overline{1.42)}$ & 118 & $\underline{1.55} \underline{(1.28-1.87)}$ \\
\hline \multicolumn{14}{|c|}{ Gastrointestinal tract } \\
\hline Gingivitis & 917 & 209 & $0.98(0.84-1.14)$ & 89 & $1.14(0.92-1.43)$ & $<11$ & $0.67(0.35-1.30)$ & 24 & $0.70(0.46-1.05)$ & 89 & $1.14(0.92-1.43)$ & $<11$ & $0.94(0.47-1.89)$ \\
\hline Gastroenteritis & 5312 & 1299 & $1.03(0.96-1.10)$ & 488 & $1.04(0.95-1.15)$ & 79 & $1.03(0.82-1.30)$ & 175 & $0.88(0.75-1.02)$ & 107 & $1.04(0.85-1.26)$ & 62 & $1.27(0.99-1.65)$ \\
\hline \multicolumn{14}{|l|}{ Urinary tract } \\
\hline Cystitis $^{b}$ & 35283 & 13876 & $1.04(1.02-1.07)$ & 3410 & $1.01(0.96-1.06)$ & 494 & $1.05(0.93-1.18)$ & 1536 & \begin{tabular}{|l|}
$1.02(0.96-1.09)$ \\
\end{tabular} & 794 & \begin{tabular}{|l|}
$1.05(0.96-1.16)$ \\
\end{tabular} & 565 & $1.11(1.00-1.23)$ \\
\hline Prostatitis $^{c}$ & 16203 & 3620 & $1.12(1.07-1.17)$ & 1194 & $1.02(0.96-1.09)$ & 256 & $1.05(0.91-1.20)$ & 555 & $1.19(1.08-1.31)$ & 246 & $1.05(0.91-1.21)$ & 128 & $0.96(0.79-1.16)$ \\
\hline Pyelonephritis $^{b}$ & 2184 & 799 & $\overline{0.95}(\overline{0.88}-\overline{1.03)}$ & 214 & $1.01(0.88-1.17)$ & 34 & $1.14(0.81-1.61)$ & 74 & $0.79(0.62-0.99)$ & 37 & $0.77(0.55-1.07)$ & 20 & $0.91(0.58-1.42)$ \\
\hline $\begin{array}{l}\text { Abbreviations: } \mathrm{Cl} \\
\text { which the numbe } \\
{ }^{\mathrm{a}} \text { ORs and } 95 \% \mathrm{Cl} \\
\mathrm{b}_{\mathrm{Females}} \text { only. } \\
{ }^{\mathrm{c}} \text { Males only. }\end{array}$ & $\begin{array}{l}\text { onfidence } \\
\text { exposed } \mathrm{p} \\
\text { ere adjuste }\end{array}$ & $\begin{array}{l}\text { interval; } \\
\text { patients is } \\
\text { d for age }\end{array}$ & $\begin{array}{l}\mathrm{VHL}=\text { non-Hodgkir } \\
\text { between } 1 \text { and } 10 \\
(66-69,70-74,75\end{array}$ & $\begin{array}{l}n \text { lymph } \\
0, \text { are lis } \\
-79,80\end{array}$ & $\begin{array}{l}\text { Ioma; } O R=\text { odds } r \\
\text { ted as '<11' to pr } \\
-84 \text { and } 85-99 \text { year }\end{array}$ & s), genc & $\begin{array}{l}\text { subjects' anonymi } \\
\text { der and diagnosis }\end{array}$ & ectior & $\begin{array}{l}\text { cordance with the } \\
\text { n year. }\end{array}$ & . & $\begin{array}{l}0.00045) \text { are } \mathrm{u} \\
\text { dicare data use }\end{array}$ & rlin & $\begin{array}{l}\text { Observations, } \\
\text { nt. }\end{array}$ \\
\hline
\end{tabular}

stimulation and/or subclinical immune deficiency, predisposing patients to both infections and lymphoma, may therefore explain the associations identified between infection-related conditions and lymphoma.

Interestingly, in the US Veterans study, associations between infections and NHL were most noticeable in those aged $<50$ years (Koshiol et al, 2011). Unfortunately, we were unable to assess these associations in a similar age group but have demonstrated, in a non-selected, population-based study, that similar associations are observed in individuals aged $\geqslant 66$ years. In stratified analyses, we substantiated the observation that associations with infectionrelated conditions, particularly sinusitis, pharyngitis, bronchitis and cellulitis, were significantly stronger in black than in white individuals (Koshiol et al, 2011). Further analysis by NHL subtypes was hindered by small numbers. Differences in immune-associated polymorphisms by race have been reported (Skibola et al, 2010) and could explain the differential effects observed.

Herpes zoster, commonly known as shingles, has previously been reported to precede HL (Tavani et al, 2000) and other haematological malignancies (Gramenzi et al, 1991; Landgren et al, 2007; Brown et al, 2008; Anderson et al, 2009; Kristinsson et al, 2010; Liu et al, 2012). Shingles is a skin rash caused by reactivation of the varicella-zoster virus, which commonly occurs in immunosuppressed individuals (Kennedy, 2002; Grulich et al, 2007).
Herpes zoster infection remained significantly associated with HL even when claims were made $>6$ years previously. Because of limited sample size, we were unable to investigate infection claims in a longer time frame preceding lymphoma diagnosis, but it has been suggested that lymphoma risk is restricted to the first 10 years since infection onset (Tavani et al, 2000). We observed similar associations between herpes zoster and DLBCL and FL, supporting previous reports in some (Karunanayake et al, 2012; Liu et al, 2012) but not all studies (Cartwright et al, 1988; Becker et al, 2012). Given the timing of these associations, it is possible that herpes zoster infection is a marker of an immunocompromised state many years before diagnosis or that it instigates a decline in cell-mediated immunity (Liu et al, 2012).

Cellulitis, a skin infection generally caused by staphylococcus or streptococcus bacteria, was associated with all NHL subtypes, particularly T-cell lymphoma. T-cell lymphomas are a heterogeneous group of lymphomas that can present with extensive skin and soft tissue necrosis, resembling an infectious process (Serra et al, 1998; Jia and Sun, 2004; Falagas et al, 2007). Misdiagnosis of T-cell lymphomas could explain the observed associations particularly as some cutaneous T-cell lymphomas are often difficult to diagnose in the early stages of disease (Serra et al, 1998; Falagas et al, 2007; Soo et al, 2011). However, the long latency period between infection and diagnosis suggests that misdiagnosis of T-cell lymphoma is unlikely 
Table 3. Main associations for non-Hodgkin lymphoma (combined) stratified by race

\begin{tabular}{|c|c|c|c|c|c|}
\hline & \multicolumn{2}{|c|}{ Whites $^{a}$} & \multicolumn{2}{|c|}{ Blacks $^{a}$} & \multirow[b]{2}{*}{ Wald $P$-value } \\
\hline Infection-related conditions by site & No. & OR $(95 \% \mathrm{Cl})$ & No. & OR $(95 \% \mathrm{Cl})$ & \\
\hline \multicolumn{6}{|l|}{ Respiratory tract } \\
\hline $\begin{array}{l}\text { Common cold } \\
\text { Sinusitis } \\
\text { Laryngitis } \\
\text { Pharyngitis } \\
\text { Bronchitis } \\
\text { Influenza } \\
\text { Pneumonia }\end{array}$ & $\begin{array}{r}1510 \\
8699 \\
1630 \\
5004 \\
10190 \\
3460 \\
6745\end{array}$ & $\frac{1.09}{\frac{1.13}{1.18}} \frac{(1.03-1.16)}{\frac{(1.11}{1.13}} \frac{(1.17)}{\left.\frac{(1.08}{1.17}-1.15\right)} \frac{(1.10}{\frac{1.17}{1.11}} \frac{(1.17)}{\frac{(1.13}{1.122)}}$ & $\begin{array}{r}67 \\
347 \\
50 \\
206 \\
385 \\
134 \\
304 \\
\end{array}$ & $\begin{array}{l}1.18(0.90-1.55) \\
\frac{1.35}{1.28} \frac{(1.18-1.55)}{(0.94-1.75)} \\
\frac{1.42}{1.22} \frac{(1.21-1.68)}{(1.07-1.38)} \\
1.13(0.93-1.37) \\
1.17(1.02-1.35)\end{array}$ & $\begin{array}{r}0.325 \\
<0.001 \\
0.178 \\
<0.001 \\
<0.001 \\
0.254 \\
0.051\end{array}$ \\
\hline \multicolumn{6}{|l|}{ Skin } \\
\hline $\begin{array}{l}\text { Cellulitis } \\
\text { Herpes zoster }\end{array}$ & $\begin{array}{l}8077 \\
2346\end{array}$ & $\frac{1.13}{1.26} \frac{(1.09-1.16)}{(1.20-1.33)}$ & $\begin{array}{r}359 \\
55\end{array}$ & $\frac{1.27}{1.32} \frac{(1.12-1.45)}{(0.98-1.78)}$ & $\begin{array}{r}<0.001 \\
0.128\end{array}$ \\
\hline \multicolumn{6}{|l|}{ Gastrointestinal tract } \\
\hline $\begin{array}{l}\text { Gingivitis } \\
\text { Gastroenteritis }\end{array}$ & $\begin{array}{r}159 \\
1152\end{array}$ & $\begin{array}{l}1.09(0.91-1.31) \\
1.04(0.97-1.12)\end{array}$ & $\begin{array}{l}12 \\
32\end{array}$ & $\begin{array}{l}0.74(0.41-1.35) \\
0.83(0.57-1.21)\end{array}$ & $\begin{array}{l}0.349 \\
0.302\end{array}$ \\
\hline \multicolumn{6}{|l|}{ Urinary tract } \\
\hline $\begin{array}{l}\text { Cystitis }^{\mathbf{b}} \\
\text { Prostatitis }^{c} \\
\text { Pyelonephritis }^{\mathbf{b}}\end{array}$ & $\begin{array}{r}12317 \\
3244 \\
703\end{array}$ & $\begin{array}{l}1.03(1.00-1.06) \\
\frac{1.10}{0.96} \frac{(1.05-1.15)}{(0.88-1.05)}\end{array}$ & $\begin{array}{r}618 \\
133 \\
37\end{array}$ & $\begin{array}{l}1.16(1.03-1.29) \\
1.18(0.96-1.44) \\
0.98(0.69-1.40)\end{array}$ & $\begin{array}{l}0.076 \\
0.054 \\
0.821\end{array}$ \\
\hline $\begin{array}{l}\text { Abbreviations: } \mathrm{Cl}=\text { confidence interval; } \mathrm{OR}=\text { odds } \\
\text { a } \mathrm{OR} \text { and } 95 \% \mathrm{Cls} \text { were adjusted for age }(66-69,7 \\
\mathrm{b}_{\text {Females only. }} \\
{ }^{c_{\text {Males only. }}}\end{array}$ & ciations & $\begin{array}{l}\text { ollowing Bonferron } \\
\text { ars), gender and }\end{array}$ & .0004 & & \\
\hline
\end{tabular}

to fully explain the observed associations, and further investigation by $\mathrm{T}$-cell subtype may provide further insight.

We did not observe any strong associations between pyelonephritis (or cystitis or prostatitis, which generally precede pyelonephritis) and NHL or associated NHL subtypes despite previous reports (La Vecchia et al, 1992; Tavani et al, 2000). However, these studies have been limited by the number of exposed cases and relied on self-reported diagnoses, potentially explaining the disparate findings (La Vecchia et al, 1992; Tavani et al, 2000). Similarly we did not find any associations between gastrointestinal infections and lymphoma.

Koshiol et al (2011) reported that viral (RR 1.6, 95\% CI 1.5-1.8) and parasitic (RR 1.7, 95\% CI 1.5-1.8) infections were more strongly associated with NHL than bacterial infections (RR 1.2, 95\% CI 1.1-1.3). Although our study did not include these broad categorisations, the vast majority of conditions associated with NHL were of viral origin, including sinusitis, influenza, bronchitis and herpes zoster.

Although we were able, for the first time, to extensively study infection-related conditions by NHL subtype, the heterogeneous nature of NHLs may mean that some associations were masked by incorporating them into these broad categories. We have previously reported associations between infection-related conditions and CLL in the SEER-Medicare data set (Anderson et al, 2009), but despite being the largest study to date, we had limited sample sizes to investigate rare or more specific NHL subtypes. The population-based sampling of cases and controls means that these findings are more representative than those from hospital-based case-control studies (La Vecchia et al, 1992; Tavani et al, 2000) or specialised registers (Doody et al, 1992; Koshiol et al, 2011) but are limited to the elderly population and by lack of lifetime exposure information to chronic or repeated infection exposure.
Exposure status was not limited by recall bias inherent in case-control studies (Cartwright et al, 1988; La Vecchia et al, 1992; Tavani et al, 2000; Chang et al, 2005; Becker et al, 2012; Karunanayake et al, 2012; Liu et al, 2012), although the use of claims data, instead of diagnostically confirmed infections, means that misclassification of exposure status is possible. This misclassification would be unlikely to be differential in nature, especially for claims many years before lymphoma diagnosis; however, overdiagnosis of infections, such as cellulitis, is possible. As both inpatient and outpatient claims were incorporated into the study, we were able to investigate a broader range of common infection-related conditions than previous studies (Cartwright et al, 1988; Doody et al, 1992; La Vecchia et al, 1992; Tavani et al, 2000; Chang et al, 2005; Koshiol et al, 2011; Karunanayake et al, 2012; Liu et al, 2012). Despite this strength, infections requiring few physician visits, such as the common cold, are likely to be underestimated. As we observed no associations between these infections and lymphoma risk, it supports the contention that we did not have differential diagnosis between cases and controls due to early prediagnostic symptoms. As diagnosis of infections were based on the attending physician claiming compensation, it was not possible to examine characteristics of infections more closely (e.g., whether herpes zoster and varicella-zoster virus-positive patients had the characteristic rash or were only antibody positive). Additionally, we were not able to differentiate the cause of the infection-related conditions and unable to comment on the severity of the infections encountered. Our models were adjusted for limited confounding variables, and hence residual confounding effects by other factors, such as comorbidities, could not be captured. Comorbidities with immune disturbance, such as autoimmune conditions which have been linked with lymphoma (Brown et al, 2008), could increase the susceptibility to infection. 


\begin{tabular}{|c|c|c|c|c|}
\hline Infection-related conditions by site & $\begin{array}{l}\text { 13-30 months } \\
\text { OR }(95 \% \mathrm{CI})^{\mathrm{a}}\end{array}$ & $\begin{array}{l}31-48 \text { months } \\
\text { OR }(95 \% \mathrm{Cl})^{a}\end{array}$ & $\begin{array}{l}49-72 \text { months } \\
\text { OR }(95 \% \mathrm{Cl})^{a}\end{array}$ & $\begin{array}{l}>72 \text { months } \\
\text { OR }(95 \% \mathrm{Cl})^{\mathrm{a}}\end{array}$ \\
\hline \multicolumn{5}{|l|}{ Non-Hodgkin lymphoma } \\
\hline $\begin{array}{l}\text { Sinusitis } \\
\text { Laryngitis } \\
\text { Pharyngitis } \\
\text { Bronchitis } \\
\text { Influenza } \\
\text { Pneumonia } \\
\text { Cellulitis } \\
\text { Herpes zoster } \\
\text { Prostatitis }\end{array}$ & $\begin{array}{l}1.11(1.05-1.16) \\
1.10(0.99-1.22) \\
1.04(0.97-1.10) \\
1.05(1.00-1.10) \\
1.26(1.20-1.32) \\
1.051 .00-1.10) \\
1.11(1.06-1.16) \\
1.29(1.19-1.40) \\
1.07(1.00-1.16)\end{array}$ & $\begin{array}{l}1.30(1.23-1.37) \\
1.34(1.20-1.49) \\
1.21(1.13-1.29) \\
1.25(1.19-1.31) \\
1.08(0.98-1.20) \\
1.18(1.12-1.25) \\
1.14(1.09-1.20) \\
1.36(1.24-1.49) \\
1.12(1.03-1.21)\end{array}$ & $\begin{array}{l}1.18(1.12-1.23) \\
1.16(1.05-1.29) \\
1.12(1.06-1.19) \\
1.15(1.10-1.19) \\
1.00(0.92-1.10) \\
1.08(1.02-1.14) \\
1.17(1.11-1.23) \\
1.24(1.13-1.36) \\
1.11(1.03-1.20)\end{array}$ & $\begin{array}{l}1.12(1.07-1.17) \\
1.13(1.01-1.26) \\
1.09(1.03-1.16) \\
1.12(1.08-1.17) \\
1.07(0.99-1.17) \\
1.14(1.08-1.20) \\
1.18(1.12-1.24) \\
1.24(1.13-1.37) \\
1.17(1.09-1.25)\end{array}$ \\
\hline \multicolumn{5}{|l|}{ Diffuse large B-cell lymphoma } \\
\hline $\begin{array}{l}\text { Sinusitis } \\
\text { Bronchitis } \\
\text { Influenza } \\
\text { Cellulitis } \\
\text { Herpes zoster }\end{array}$ & $\begin{array}{l}1.09(1.01-1.18) \\
1.04(0.97-1.11) \\
1.21(1.11-1.31) \\
1.05(0.98-1.13) \\
1.13(0.99-1.29)\end{array}$ & $\begin{array}{l}1.28(1.19-1.39) \\
1.15(1.07-1.24) \\
0.98(0.84-1.14) \\
1.08(1.00-1.17) \\
1.26(1.09-1.46)\end{array}$ & $\begin{array}{l}1.15(1.07-1.23) \\
1.11(1.04-1.19) \\
1.04(0.91-1.18) \\
1.13(1.05-1.22) \\
1.28(1.11-1.46)\end{array}$ & $\begin{array}{l}1.08(1.00-1.15) \\
1.13(1.06-1.21) \\
1.07(0.94-1.21) \\
1.12(1.03-1.21) \\
1.16(1.00-1.35)\end{array}$ \\
\hline \multicolumn{5}{|l|}{ T-cell NHL } \\
\hline $\begin{array}{l}\text { Pharyngitis } \\
\text { Cellulitis }\end{array}$ & $\begin{array}{l}1.24(1.01-1.52) \\
1.33(1.14-1.56)\end{array}$ & $\begin{array}{l}1.26(1.00-1.57) \\
1.42(1.20-1.67)\end{array}$ & $\begin{array}{l}1.34(1.10-1.62) \\
1.41(1.20-1.66)\end{array}$ & $\begin{array}{l}1.17(0.96-1.43) \\
1.29(1.08-1.54)\end{array}$ \\
\hline \multicolumn{5}{|l|}{ Follicular lymphoma } \\
\hline $\begin{array}{l}\text { Sinusitis } \\
\text { Laryngitis } \\
\text { Herpes zoster }\end{array}$ & $\begin{array}{l}1.10(0.99-1.22) \\
1.14(0.90-1.43) \\
1.40(1.18-1.68)\end{array}$ & $\begin{array}{l}1.35(1.21-1.51) \\
1.25(0.97-1.60) \\
1.24(1.00-1.53)\end{array}$ & $\begin{array}{l}1.10(0.99-1.22) \\
1.30(1.04-1.63) \\
1.02(0.81-1.28)\end{array}$ & $\begin{array}{l}1.16(1.05-1.29) \\
1.33(1.06-1.67) \\
1.25(1.00-1.55)\end{array}$ \\
\hline \multicolumn{5}{|l|}{ Marginal zone lymphoma } \\
\hline $\begin{array}{l}\text { Sinusitis } \\
\text { Bronchitis } \\
\text { Influenza } \\
\text { Pneumonia } \\
\text { Cellulitis }\end{array}$ & $\begin{array}{l}1.50(1.30-1.72) \\
1.13(0.98-1.30) \\
1.37(1.18-1.60) \\
1.25(1.07-1.46) \\
1.29(1.12-1.50)\end{array}$ & $\begin{array}{l}1.34(1.14-1.57) \\
1.23(1.06-1.43) \\
1.53(1.17-2.01) \\
1.32(1.11-1.57) \\
1.23(1.05-1.44)\end{array}$ & $\begin{array}{l}1.32(1.15-1.51) \\
1.21(1.07-1.38) \\
1.18(0.91-1.52) \\
1.05(0.88-1.25) \\
1.24(1.07-1.44)\end{array}$ & $\begin{array}{l}1.16(1.01-1.33) \\
1.07(0.94-1.23) \\
1.25(0.99-1.59) \\
1.27(1.08-1.49) \\
1.16(0.99-1.35)\end{array}$ \\
\hline \multicolumn{5}{|l|}{ Hodgkin lymphoma } \\
\hline Herpes zoster & $1.36(0.95-1.94)$ & $1.48(1.00-2.19)$ & $1.93(1.38-2.70)$ & $1.49(1.00-2.22)$ \\
\hline
\end{tabular}

Similarly, we did not have data on characteristics and behaviours like smoking, drinking and obesity and therefore could not adjust for these factors. Finally, as we investigated numerous associations between infection-related conditions and lymphomas, some of the associations may have occurred by chance. We therefore focussed our discussion on those associations that remained after crude adjustment for multiple comparisons.

It is possible that an infection or other antigen could lead to different clinical manifestations depending on the hosts immune systems. For example, EBV is an extremely common infection that does not lead to cancer in most people. In a small subset, EBV may contribute to DLBCL (Kinch et al, 2013; Ozsan et al, 2013), whereas in most people it does not. Similarly, not all DLBCL cases are EBV-positive, reflecting heterogeneity in the aetiology even of the same NHL subtype. Immune differences resulting in different clinical outcomes could, for example, be driven by differences in HLA polymorphisms, which can affect antigen presentation.

In conclusion, several common infection-related conditions were associated with NHLs but not with HL, where only herpes zoster was more common in cases than in controls after adjustment for multiple comparisons. Herpes zoster showed the strongest associations for both NHL and HL. Most respiratory tract infections were associated with NHL, particularly MZL. Several infection-related conditions were more strongly associated with NHL in blacks than in whites. Precise elucidation of the mechanisms underlying lympho-proliferations may provide important clues for understanding how immune disturbance contributes to the development of both NHL and HL.

\section{ACKNOWLEDGEMENTS}

This work was supported by the Intramural Research Program of the National Cancer Institute. GT is a $\mathrm{PhD}$ student at the Queen's University Belfast in receipt of funding from MPD Voice. CMS is a PhD student at the Queen's University Belfast in receipt of a Department for Employment and Learning (Northern Ireland) funded scholarship. This study used the linked SEER-Medicare database. The interpretation and reporting of these data are the sole responsibility of the authors. We 
acknowledge the efforts of the Applied Research Program, NCI; the Office of Research, Development and Information, Centers for Medicare and Medicaid Services; Information Management Services, Inc.; and the Surveillance, Epidemiology, and End Results (SEER) Program tumour registries in the creation of the SEER-Medicare database. We also thank Dr Winnie Ricker, Information Management Services, Rockville, MD, USA for constructing and analysing the data set.

\section{CONFLICT OF INTEREST}

The authors declare no conflict of interest.

\section{REFERENCES}

Anderson LA, Landgren O, Engels EA (2009) Common community acquired infections and subsequent risk of chronic lymphocytic leukaemia. Br J Haematol 147: 444-449.

Anderson LA, Pfeiffer R, Warren JL, Landgren O, Gadalla S, Berndt SI, Ricker W, Parsons R, Wheeler W, Engels EA (2008) Hematopoietic malignancies associated with viral and alcoholic hepatitis. Cancer Epidemiol Biomarkers Prev 17: 3069-3075.

Becker N, Falster MO, Vajdic CM, de Sanjose S, Martínez-Maza O, Bracci PM, Melbye M, Smedby KE, Engels EA, Turner J, Vineis P, Costantini AS, Holly EA, Spinelli JJ, La Vecchia C, Zheng T, Chiu BC-H, Montella M, Cocco P, Maynadié M, Foretova L, Staines A, Brennan P, Davis S, Severson R, Cerhan JR, Breen EC, Birmann B, Cozen W, Grulich AE, Newton R (2012) Self-reported history of infections and the risk of non-Hodgkin lymphoma: an InterLymph pooled analysis. Int J Cancer 131: $2342-2348$

Brown LM, Gridley G, Check D, Landgren O (2008) Risk of multiple myeloma and monoclonal gammopathy of undetermined significance among white and black male United States veterans with prior autoimmune, infectious, inflammatory, and allergic disorders. Blood 111: 3388-3394.

Cartwright RA, McKinney PA, O’Brien C, Richards ID, Roberts B, Lauder I, Darwin CM, Bernard SM, Bird CC (1988) Non-Hodgkin's lymphoma: case control epidemiological study in Yorkshire. Leuk Res 12: 81-88.

Cerhan JR, Ansell SM, Fredericksen ZS, Kay NE, Liebow M, Call TG, Dogan A, Cunningham JM, Wang AH, Liu-Mares W, Macon WR, Jelinek D, Witzig TE, Habermann TM, Slager SL (2007) Genetic variation in 1253 immune and inflammation genes and risk of non-Hodgkin lymphoma. Blood 110: 4455-4463.

Chang ET, Smedby KE, Hjalgrim H, Schöllkopf C, Porwit-MacDonald A, Sundström C, Tani E, d'Amore F, Melbye M, Adami H-O, Glimelius B (2005) Medication use and risk of non-Hodgkin's lymphoma. Am J Epidemiol 162: 965-974.

Doody MM, Linet MS, Glass AG, Friedman GD, Pottern LM, Boice JD, Fraumeni JF (1992) Leukemia, lymphoma, and multiple myeloma following selected medical conditions. Cancer Causes Control 3: 449-456.

Engels EA, Chatterjee N, Cerhan JR, Davis S, Cozen W, Severson RK, Whitby D, Colt JS, Hartge P (2004) Hepatitis C virus infection and non-Hodgkin lymphoma: results of the NCI-SEER multi-center case-control study. Int J Cancer 111: 76-80.

Engels EA, Pfeiffer RM, Ricker W, Wheeler W, Parsons R, Warren JL (2011) Use of surveillance, epidemiology, and end results-medicare data to conduct case-control studies of cancer among the US elderly. Am J Epidemiol 174: 860-870.

Falagas ME, Bliziotis IA, Rafailidis PI, Peppas G (2007) Peripheral T-cell lymphoma masquerading as infectious cellulitis. Eur J Dermatol 17: 166-167.

Gramenzi A, Buttino I, D’Avanzo B, Negri E, Franceschi S, La Vecchia C (1991) Medical history and the risk of multiple myeloma. Br J Cancer 63: 769-772.

Grulich AE, van Leeuwen MT, Falster MO, Vajdic CM (2007) Incidence of cancers in people with HIV/AIDS compared with immunosuppressed transplant recipients: a meta-analysis. Lancet 370: 59-67.

Hjalgrim H, Engels EA (2008) Infectious aetiology of Hodgkin and nonHodgkin lymphomas: a review of the epidemiological evidence. J Intern Med 264: 537-548.
Hu W, Bassig BA, Xu J, Zheng T, Zhang Y, Berndt SI, Holford TR, Hosgood HD, Leaderer B, Yeager M, Menashe I, Boyle P, Zou K, Zhu Y, Chanock S, Lan Q, Rothman N (2013) Polymorphisms in patternrecognition genes in the innate immunity system and risk of non-Hodgkin lymphoma. Environ Mol Mutagen 54: 72-77.

Jia H, Sun T (2004) Extranodal NK/T-cell lymphoma mimicking cellulitis. Leuk Lymphoma 45: 1467-1470.

Karunanayake CP, Spinelli JJ, McLaughlin JR, Dosman JA, Pahwa P, McDuffie HH (2012) Hodgkin lymphoma and pesticides exposure in men: a Canadian case-control study. J Agromedicine 17: 30-39.

Kennedy PGE (2002) Key issues in varicella-zoster virus latency. J Neurovirol 8: $80-84$.

Kinch A, Baecklund E, Backlin C, Ekman T, Molin D, Tufveson G, Fernberg P, Sundström C, Pauksens K, Enblad G (2013) A populationbased study of 135 lymphomas after solid organ transplantation: The role of Epstein-Barr virus, hepatitis C and diffuse large B-cell lymphoma subtype in clinical presentation and survival. Acta Oncol; e-pub ahead of print 28 October 2013.

Koshiol J, Lam TK, Gridley G, Check D, Brown LM, Landgren O (2011) Racial differences in chronic immune stimulatory conditions and risk of non-Hodgkin's lymphoma in veterans from the United States. J Clin Oncol 29: 378-385.

Kristinsson SY, Koshiol J, Björkholm M, Goldin LR, McMaster ML, Turesson I, Landgren O (2010) Immune-related and inflammatory conditions and risk of lymphoplasmacytic lymphoma or Waldenstrom macroglobulinemia. J Natl Cancer Inst 102: 557-567.

Landgren O, Gridley G, Check D, Caporaso NE, Morris Brown L (2007) Acquired immune-related and inflammatory conditions and subsequent chronic lymphocytic leukaemia. Br J Haematol 139: 791-798.

La Vecchia C, Negri E, Franceschi S (1992) Medical history and the risk of non-Hodgkin's lymphomas. Cancer Epidemiol Biomarkers Prev 1: 533-536.

Liu Y-C, Yang Y-H, Hsiao H-H, Yang W-C, Liu T-C, Chang C-S, Yang M-Y, Lin P-M, Hsu J-F, Chang P-Y, Lin S-F (2012) Herpes zoster is associated with an increased risk of subsequent lymphoid malignancies-a nationwide population-based matched-control study in Taiwan. $B M C$ Cancer 12: 503.

Ozsan N, Cagirgan S, Saydam G, Gunes A, Hekimgil M (2013) Epstein-Barr virus (EBV) positive diffuse large B cell lymphoma of the elderly-experience of a single center from Turkey. Pathol Res Pract 209: 471-478.

Richardson PG, Laubach J, Mitsiades CS, Schlossman RL, Ghobrial IM, Munshi NC, Anderson KC (2011) MGUS and smoldering myeloma: the most prevalent of plasma cell dyscrasias. Oncology (Williston Park) 25 : 594596.

Roulland S, Navarro J-M, Grenot P, Milili M, Agopian J, Montpellier B, Gauduchon P, Lebailly P, Schiff C, Nadel B (2006) Follicular lymphoma-like B cells in healthy individuals: a novel intermediate step in early lymphomagenesis. J Exp Med 203: 2425-2431.

Serra A, Estrach MT, Martí R, Villamor N, Rafel M, Montserrat E (1998) Cutaneous involvement as the first manifestation in a case of T-cell prolymphocytic leukaemia. Acta Derm Venereol 78: 198-200.

Serraino D, Franceschi S, Talamini R, Barra S, Negri E, Carbone A, La Vecchia C (1991) Socio-economic indicators, infectious diseases and Hodgkin's disease. Int J Cancer 47: 352-357.

Skibola CF, Bracci PM, Nieters A, Brooks-Wilson A, de Sanjosé S, Hughes AM, Cerhan JR, Skibola DR, Purdue M, Kane E, Lan Q, Foretova L, Schenk M, Spinelli JJ, Slager SL, De Roos AJ, Smith MT, Roman E, Cozen W, Boffetta P, Kricker A, Zheng T, Lightfoot T, Cocco P, Benavente Y, Zhang Y, Hartge P, Linet MS, Becker N, Brennan P, Zhang L, Armstrong B, Smith A, Shiao R, Novak AJ, Maynadie M, Chanock SJ, Staines A, Holford TR, Holly EA, Rothman N, Wang SS (2010) Tumor necrosis factor (TNF) and lymphotoxin-alpha (LTA) polymorphisms and risk of non-Hodgkin lymphoma in the InterLymph Consortium. Am J Epidemiol 171: 267-276.

Soo KL, Shustik D, Yusoff LZM, Tan L, Tan SY (2011) An algorithmic approach to the diagnosis of NK and $\mathrm{T}$ cell lymphomas. Pathology 43: 673-681.

Tavani A, La Vecchia C, Franceschi S, Serraino D, Carbone A (2000) Medical history and risk of Hodgkin's and non-Hodgkin's lymphomas. Eur J Cancer Prev 9: 59-64. 
Turner JJ, Morton LM, Linet MS, Clarke CA, Kadin ME, Vajdic CM, Monnereau A, Maynadié M, Chiu BC-H, Marcos-Gragera R, Costantini AS, Cerhan JR, Weisenburger DD (2010) InterLymph hierarchical classification of lymphoid neoplasms for epidemiologic research based on the WHO classification (2008): update and future directions. Blood 116: e90-e98.

Warren JL, Klabunde CN, Schrag D, Bach PB, Riley GF (2002) Overview of the SEER-Medicare data: content, research applications, and generalizability to the United States elderly population. Med Care 40: IV-3-18.

This work is published under the standard license to publish agreement. After 12 months the work will become freely available and the license terms will switch to a Creative Commons AttributionNonCommercial-Share Alike 3.0 Unported License. 